\title{
Aktuelle Studien zur Wirksamkeit künstlerischer Therapien auf emotionale und soziale Parameter, Lebensqualität und Wohlbefinden
}

\author{
Iris Bräuninger
}

\section{Einführung}

D ieser Beitrag präsentiert die Ergebnisse eines Systematischen Reviews und eines RCTs zur Wirksamkeit künstlerischer Therapieinterventionen auf kindbezogene Ergebnisse wie emotionale und soziale Parameter, Lebensqualität und Wohlbefinden von Grundschülerlnnen.

\section{Systematischer Review kindbezogener}

Ergebnisse und Bewertungen von

\section{künstlerischen Therapien}

Moula, Aithal, Karkou und Powell (2020) überprüften in ihrem Systematischen Review, wie Grundschulkinder (5-12 Jahre) ihre Teilnahme an künstlerischen Therapien anhand von sogenannten PROMs (participant-reported outcome measures) bewerteten. Die Autorlnnen führten ihre Systematische Recherche in den elektronischen Datenbanken AMED, PsycINFO, CINAHL, ERIC, MEDLINE, Campbell Collaboration Library, WHO ICTRP, Cochrane durch. Zusätzlich schlossen sie relevante graue Literatur, Zeitschriften für Künstlerische Therapien und ExpertInnen-Informationen in ihre Suche ein. Direkt berichtete Ergebnisse aus qualita- tiven (z. B. Interviews) und quantitativen Erhebungen zu musiktherapeutischen, kunsttherapeutischen, tanz-, bewegungstherapeutischen und dramatherapeutischen Interventionen wurden als Einschlusskriterien definiert. Ausgeschlossen wurden Studien, welche diese Kriterien nicht erfüllten, z. B. bezüglich Population ( 5 resp. >12 Jahre), bezüglich Interventionen (künstlerische Therapien in Kombination mit anderen Therapien - außer eine Subgruppenanalyse lag vor), bezüglich fehlendem Outcome-Reporting oder bezüglich Studiendesign (Fallbesprechungen, Reviews, lückenhaftes Design, nicht englische Publikation etc.).

Von 11.362 Studien wurden 9.214 Publikationen gescreent, wovon 237 Volltextartikel ausgewertet wurden. Letztendlich gingen sieben Artikel in die Systematische Analyse ein (davon sechs quantitative und eine qualitative Studie): zwei Pilot-RCTs, zwei Quasi-RCTs, ein ClusterRCT, eine kontrollierte Studie mit Prä-Test- / Post-Test-Design und eine Studie mit fundiertem Theorie-Design. Drei davon waren Musiktherapiestudien und je zwei Kunsttherapie-, und Tanz-, Bewegungstherapiestudien (keine Dramatherapiestudie). Der Interventionszeitraum variierte zwischen 8 und 20 Therapieeinheiten, die Therapieeinheiten betrugen zwi- 
schen 45 und 120 Minuten und fanden ein- bis dreimal wöchentlich statt. Insgesamt nahmen 358 Teilnehmerlnnen an den sieben Studien teil. Die von den Kindern berichteten signifikanten PROMS bezogen sich auf Selbstwertgefühl, Selbstvertrauen, Selbstausdruck, Stimmung, Kommunikation, Verständnis, Belastbarkeit, Lernen und aggressives Verhalten. Sie berichteten ferner über kleine positive Veränderungen von Depressionen, Angstzuständen, Aufmerksamkeitsproblemen und zurückgezogenem Verhalten.

\section{Ein RCT zur Wirksamkeit künstlerischer Therapien auf die Lebensqualität und das Wohlbefinden in Grundschulen}

Moula, Powell und Karkou (2020) überprüften in einem RCT die Wirksamkeit von künstlerischen Therapien in Grundschulen. Kinder und LehrerInnen bewerteten als Outcome-Variablen die gesundheitsbezogene Lebensqualität (HRQOL-Skala; EQ), das Wohlbefinden und die Lebensfunktion (CORS), emotionale und Verhaltensstörungen (SDQ) und Schlafdauer (Fitbits). Die Pilotstudie wurde als RCT-CrossOver-Pilotdesign durchgeführt und diente zur Kalkulation der Stichprobengröße für zukünftige groß angelegte Studien. An der Studie nahmen 62 Kinder (7-10 Jahre) mit leichten emotionalen und Verhaltensstörungen teil (Kunsttherapie $n=14$, Tanz-, Bewegungstherapie $n=16$, Musiktherapie $n=14$, Dramatherapie $n=12$ ); 31 wurden auf die Interventionsgruppe und 31 auf die anfängliche Warte-Kontrollgruppe randomisiert. Die Baseline-Tests wurden in beiden Gruppen parallel durchgeführt. Der Prä-Test wurde in der Interventionsgruppe vor der achtwöchigen Intervention durchgeführt. Der Post-Test wurde in der Interventionsgruppe nach der achtwöchigen Intervention durchgeführt. Zum gleichen Zeitpunkt wurde die Warte-Kontrollgruppe zur Interventionsgruppe, und der PräTest wurde durchgeführt, nach achtwöchiger
Intervention folgte dann der Post-Test. In beiden Gruppen folgten die Follow-Up-Tests je 3, 6 und 12 Monate nach der Intervention. Signifikante Verbesserungen zeigten sich für die Schlafdauer $(P=0,002)$ und bei den emotionalen und Verhaltensstörungen (SDQ) $(P=0,008)$. Keine signifikanten Verbesserungen ergaben sich für die gesundheitsbezogene Lebensqualität (HRQOL) und das Wohlbefinden (CORS). Für zukünftige Studien seien alle Zielparameter geeignet, und 225 Teilnehmerlnnen wären als Stichprobengröße für die erforderliche Effektstärke notwendig.

\section{Schlussfolgerung}

Die Autorinnen des Systematischen Reviews und des RCTs betonten, dass der langfristige Nachweis der künstlerischen Therapien bei Grundschulkindern noch ausstehe. Die Heterogenität der bislang berichteten Forschungsergebnisse weise auf die Notwendigkeit hin, dass sich zukünftige Wirksamkeitsforschung von Therapien mit Kindern auf die Durchführung von Therapieeinheiten konzentrieren solle, die theoretisch fundiert und für die spezifische Population geeignet seien. Ferner solle sie standardisierten Richtlinien für die Berichterstattung folgen und in Hinblick auf die Assessments und Zielparameter von Kindern validiert worden sein.

Die Ergebnisse des RCTs wiesen auf Verbesserungen der Schlafdauer und der emotionalen und Verhaltensstörungen bei Grundschulkindern durch künstlerische Therapien hin. Die nicht signifikante Verbesserung der Lebensqualität (HRQOL) und des Wohlbefindens könnte eventuell Ergebnis des Designs sein, das keinen direkten Between-Group-Vergleich vorsah. Zukünftige große Studien sollten diesen Vergleich durchführen. Zukünftige Wirksamkeitsstudien mit Kindern könnten außerdem vermehrt die Bewertungen durch Kinder in Outcome-Messungen berücksichtigen. 


\section{Literatur}

Moula, Z., Aithal, S., Karkou, V., Powell, J. (2020): A systematic review of child-focused outcomes and assessments of arts therapies delivered in primary mainstream schools. Children and Youth Services Review 112, https://doi.org/ 10.1016/j.childyouth.2020.104928

Moula, Z., Powell, J., Karkou, V. (2020): An investigation of the effectiveness of arts therapies interventions on measures of quality of life and wellbeing: a pilot randomized controlled study in primary schools. Frontiers in Psychology 11, 3591, https://doi.org/10.3389/fpsyg.2020. 586134

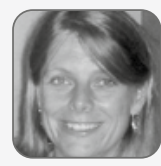

\section{Dr. Iris Bräuninger}

Senior Researcher \& Co-Leiterin Studiengang Psychomotoriktherapie (Hochschule für Heilpädagogik Zürich IVE), Dozentin MA Tanztherapie UAB Barcelona, BTD-Supervisorin / Ausbilderin / Lehrtherapeutin, KMP-Notatorin, Praxis Tanztherapie Supervision Bodensee.

Dr. Iris Bräuninger dancetherapy@mac.com und iris.braeuninger@hfh.ch 